\title{
Using an activity tracker to increase motivation for physical activity in patients with type 2 diabetes in primary care: a randomized pilot trial
}

\author{
Cynthia Pelletier ${ }^{1,2} \wedge$, Marie-Pierre Gagnon ${ }^{2,3}$, Natalie Alméras ${ }^{4,5}$, Jean-Pierre Després ${ }^{2,4,5}$, Paul Poirier ${ }^{4,6}$, \\ Angelo Tremblay ${ }^{2,4,5}$, Christian Chabot $^{2}$, Caroline Rhéaume ${ }^{1,2,4} \wedge$
}

${ }^{1}$ Department of Family Medicine and Emergency Medicine, Faculty of Medicine, Université Laval, Québec, Canada; ${ }^{2}$ VITAM - Centre de recherche en santé durable, CIUSSS-Capitale-Nationale, Université Laval, Québec, Canada; ${ }^{3}$ Faculty of Nursing, Université Laval, Québec, Canada; ${ }^{4}$ Centre de recherche de l'Institut universitaire de cardiologie et de pneumologie de Québec, Québec, Canada; ${ }^{5}$ Department of Kinesiology, Faculty of Medicine, Université Laval, Québec, Canada; ${ }^{6}$ Faculty of Pharmacy, Université Laval, Québec, Canada

Contributions: (I) Conception and design: MP Gagnon, C Chabot, C Rhéaume; (II) Administrative support: MP Gagnon, C Rhéaume, C Chabot, N Alméras; (III) Provision of study materials or patients: MP Gagnon, C Rhéaume; (IV) Collection and assembly of data: C Pelletier, MP Gagnon, C Rhéaume; (V) Data analysis and interpretation: C Pelletier, MP Gagnon, C Rhéaume; (VI) Manuscript writing: All authors; (VII) Final approval of manuscript: All authors.

Correspondence to: Caroline Rhéaume, MD, PhD. Department of Family Medicine and Emergency Medicine, Faculty of Medicine, Université Laval, 1050 avenue de la Médecine, Pavillon Ferdinand-Vandry, local 4617, Québec (QC), G1V 0A6, Canada. Email: caroline.rheaume@fmed.ulaval.ca.

Background: Adopting healthy lifestyle habits reduces the risk of type 2 diabetes (T2D) and its complications. The use of an activity tracker to monitor physical activity (PA) could favor behavior changes in patients with chronic diseases such as diabetes. The aims of this study were: (I) to evaluate the impact of an activity tracker on PA and cardiometabolic risk variables in patients with T2D; (II) to assess the feasibility of its implantation in a primary care setting.

Methods: This 3-month study was a pilot randomized controlled trial of 30 patients with T2D followed at a university-affiliated Family Medicine Group. Patients were randomly assigned to either: (I) control group, including a PA promotion intervention supported by a kinesiologist or (II) intervention group, including a PA promotion intervention supported by a kinesiologist with the addition of an activity tracker (Fitbit). Cardiometabolic risk variables, PA and motivation were assessed at baseline and after three months. Satisfaction and acceptability of wearing the activity tracker were measured in the intervention group.

Results: PA assessed by questionnaires increased in both groups, change being greater in the intervention group $(\mathrm{P}<0.05)$. Autonomous motivation in both groups was higher than controlled motivation $(\mathrm{P}<0.001)$. Eighty-six percent of the participants in the intervention group were satisfied with their activity tracker use and the compliance remained high. High-density lipoprotein cholesterol increased in the intervention group and decreased in the control group $(\mathrm{P}=0.014)$. Resting systolic and diastolic blood pressure decreased over time in both groups $(\mathrm{P}<0.05)$ whereas glycated hemoglobin tended to decrease in both groups $(\mathrm{P}=0.080)$. Significant correlations were observed between average steps per day and changes in waist circumference (pre: -0.721, $\mathrm{P}=0.044$; post: $-0.736, \mathrm{P}=0.038$ ), body mass index (pre: $-0.764, \mathrm{P}=0.010$; post: $-0.771, \mathrm{P}=0.009$ ) and fat percentage (pre: $-0.654, \mathrm{P}=0.040$; post: $-0.686, \mathrm{P}=0.028$ ) in the intervention group.

Conclusions: Our pilot study shows that the use of an activity tracker improves cardiometabolic risk variables in patients with T2D and could potentially be a motivation tool to increase PA in primary care setting.

^ ORCID: Cynthia Pelletier, 0000-0003-1573-0519; Caroline Rhéaume, 0000-0002-1863-4410. 
Keywords: Activity tracker; type 2 diabetes (T2D); physical activity (PA); motivation behavior

Received: 06 November 2020; Accepted: 07 February 2021; Published: 20 October 2021.

doi: $10.21037 /$ mhealth-20-154

View this article at: http://dx.doi.org/10.21037/mhealth-20-154

\section{Introduction}

According to the World Health Organization, diabetes is the direct cause of 1.6 million deaths each year (1). The International Diabetes Federation estimates that 463 million adults were living with diabetes in 2019 worldwide and this number may increase up to 700 million in 2045 (2). Half of the global diabetes spending occurs in North America and the Caribbean (2). The direct and indirect costs are estimated to 3 billion dollars in the province of Québec (3).

Physical inactivity contributes to premature mortality, coronary heart disease and type 2 diabetes (T2D), impacting health similarly to smoking or obesity (4). It is recognized that the adoption of healthy lifestyle habits, such as the regular practice of physical activity (PA), contributes to significantly reduce the prevalence of T2D and its complications $(5,6)$. A lifestyle intervention including healthy diet and regular PA in patients with diabetes can reduce weight, glycated hemoglobin and cardiovascular risk factors (7-9). Regardless of the training modality, exercise can improve quality of life in patients with T2D (10). In the province of Québec, only $41 \%$ of the population aged $\geq 15$ years old meets PA recommendations (11). Many technological approaches have been developed in order to try to improve PA and health such as mobile applications (apps) (12-15), pedometers (16) and activity trackers $(17,18)$ to contribute to the adoption of healthier behaviors.

Fitbit (Fitbit Inc, San Francisco, CA) and Jawbone (Jawbone Inc, San Francisco, CA) are two popular brands of activity trackers. Systematic reviews indicate that they present a high validity for steps, but a low validity regarding energy expenditure and sleep (19-21). In chronic diseases such as T2D, systematic reviews (22-25) and randomized controlled trials (26-29) document that pedometers and activity trackers can contribute to significantly increase PA.

To the best of our knowledge, no study in primary care setting have assessed the use of an activity trackerbased intervention supported by a kinesiologist to increase motivation regarding $\mathrm{PA}$ in patients with $\mathrm{T} 2 \mathrm{D}$. The goals of this study were: (I) to evaluate the impact of an activity tracker on PA and cardiometabolic risk variables; (II) to assess the feasibility of implementing an activity tracker in patients with T2D in order to increase their motivation in a primary care setting. We tested the hypotheses that PA would be increased in the intervention group using an activity tracker with concomitant favorable cardiometabolic risk variable changes. We also wanted to verify whether the implantation of an activity tracker would be feasible in a primary care setting. We present the following article in accordance with the CONSORT reporting checklist (available at http://dx.doi.org/10.21037/mhealth-20-154).

\section{Methods}

\section{Study design}

This study was a pilot controlled trial of 30 patients randomized into two groups (Figure 1) (30-32). Sample size was determined accordingly to the availability of the equipment and the study funding. Thirty participants were randomly assigned (1:1) in each group by a statistician using a computer random number generator. Assignment was performed by the research professional following the statistician's instructions. Due to the nature of the intervention, participants and health care providers could not be blinded. The statistician who performed statistical analyses was blinded to participants' group. The control group received a PA promotion intervention supported by a kinesiologist. The intervention group also had a PA promotion intervention supported by a kinesiologist while being given an activity tracker to wear (Fitbit Charge HR, Fitbit Inc, San Francisco, CA). The intervention of the kinesiologist was necessary to optimize the activity tracker use with a PA promotion intervention and for the questionnaires completion and interpretation. In order to assess the impact of the activity tracker per see, the kinesiologist intervention was implemented in both group. Study duration was 3 months. PA, PA motivation and cardiometabolic risk variables were assessed at baseline and at 3 months. 
Recruitment:

University family medicine group

1 st

appointment

- Medical evaluation and eligibility by the clinical researcher

- Cardiometabolic measurements

- Anthropometric: weight, body mass index, waist circumference, fat \%

- Hemodynamic: blood pressure, heart rate

- Metabolic: blood tests (glycated hemoglobin, lipid profile,kidney function,

complete blood count), urine tests (albumin-creatinine ratio, microscopic urinalysis)

- Study protocol presentation and informed consent by the research professionnal

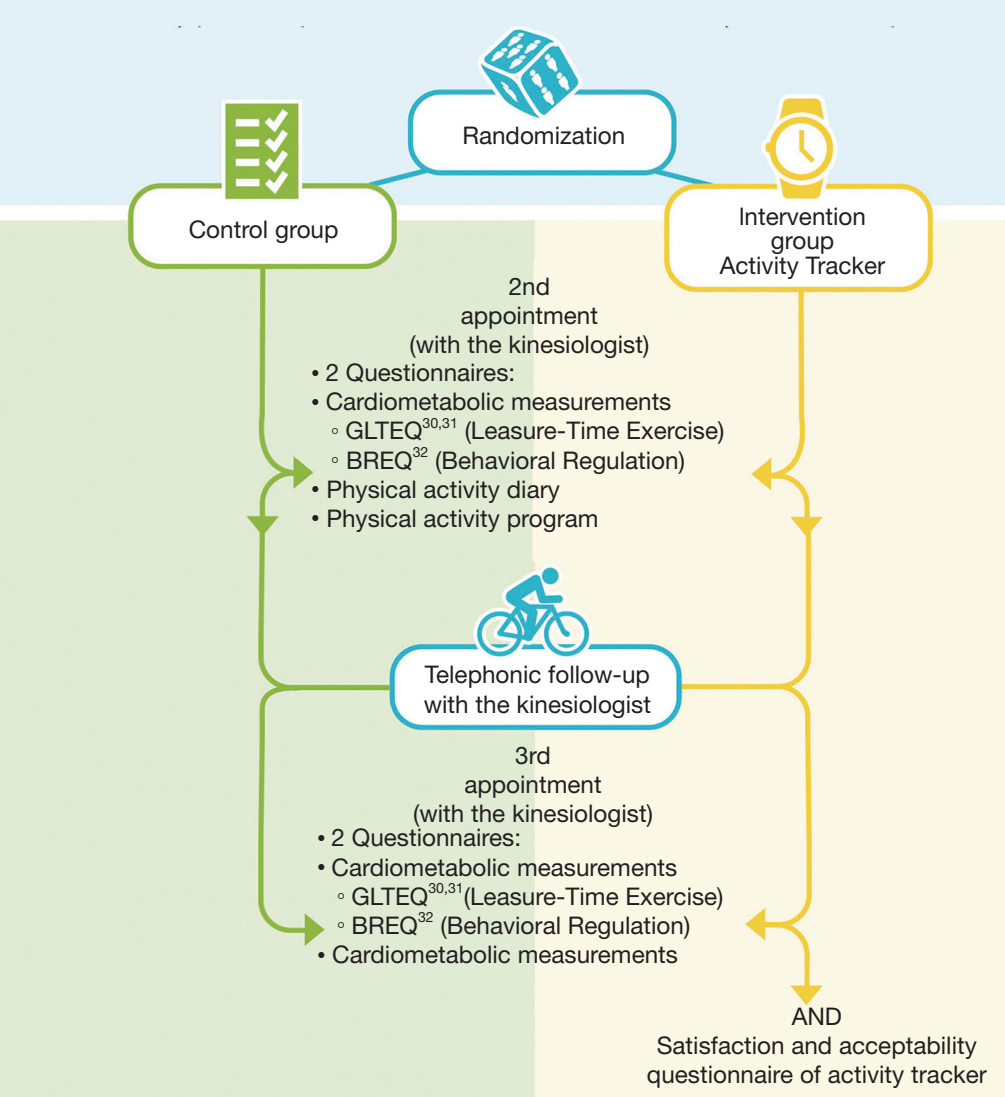

Figure 1 Illustration of pilot randomized study protocol. GLTEQ, Godin Leisure-Time Exercise Questionnaire (30,31); BREQ, Behavioral Regulation in Exercise Questionnaire version 2 (32).

\section{Participants and recruitment}

Participants had to meet the following criteria: (I) T2D man or woman between 18 and 90 years of age, (II) stable medical condition and (III) $\mathrm{PA}<150 \mathrm{~min} / \mathrm{week}$, according to the Canadian Society for Exercise Physiology (33). Patients with acute renal failure or glomerular filtration rate $<30 \mathrm{~mL} / \mathrm{min} / 1.73 \mathrm{~m}^{2}$ were excluded. All patients were followed by health professionals from a university-affiliated
Family Medicine Group (GMF-U Quatre-Bourgeois). Eligible participants were recruited by their family doctor, were informed that a study was taking place and were invited to meet the research professional.

\section{Ethical consideration}

The authors are accountable for all aspects of the work. The trial was conducted in accordance with the Declaration of 
Helsinki (as revised in 2013) (34). The study was approved by institutional ethics board of Centre intégré universitaire en santé et services sociaux (CIUSSS) de la CapitaleNationale (NO.: 2017-2018-07) and informed consent was taken from all the participants. The complete study protocol was published in ClinicalTrials.gov (NCT03709966) (35).

\section{Material}

Fitbit Charge HR and iPad (Apple Inc, Cupertino, CA) devices were provided free of charge to the patients through an infrastructure funding (Canadian Foundation for Innovation). The Fitbit device is similar to a wrist-sports watch with many features including step calculation, distance traveled, calories burned, heart rate and sleep status. The information recorded by the watch can be synchronized and transmitted directly to the Fitbit application that was installed on the iPad, allowing it to be viewed and tracked on a daily basis. Weekly and monthly reports can be produced and it is also possible to adjust personal goals.

\section{Intervention}

The intervention lasted 3 months and involved a total of 3 face-to-face appointments. The first appointment involved the physician in charge of the study in order to evaluate the patients' records and to validate their eligibility. The second appointment was attended approximately two to four weeks later with a kinesiologist who proposed a personalized PA program to all participants, consisting of 150 minutes of moderate aerobic activity and one session of muscular exercise. For those in the intervention group, a Fitbit watch and iPad with the Fitbit app were provided. At the $6^{\text {th }}$ week of the intervention, the kinesiologist had a follow-up call with all participants regarding the integration of the PA program. A third appointment was planned at the end of the intervention with the kinesiologist. Participants had to bring back their material during this appointment.

\section{Outcome measures}

\section{PA measurements}

PA was assessed in two different ways. First, participants in both groups filled a PA questionnaire and a logbook, where they auto-reported the intensity, nature and duration of their physical activity. The Godin Leisure-Time Exercise Questionnaire was used (30,31), which contains 4 items: (I) how many periods a week a strenuous exercise, a moderate exercise and a mild exercise were practiced for $>15$ minutes and (II) at which frequency (often, sometimes or never/rarely) a regular training provoked sweat/faster heart rate was performed $(30,31)$. Participants filled the questionnaire at the beginning and at the end of the study. This questionnaire in its original French version has been validated and used in many PA studies $(30,31,36,37)$. A weekly leisure-time activity score was calculated by multiplying the number of periods of strenuous, moderate and mild exercise respectively by 9,5 and 3 respectively $(30,31)$. A score of $\geq 24$ units was rated as "active", a score between 14 and 23 units as "moderately active" and a score $<14$ units as "insufficiently active" (30,31). A score related to health was also calculated by using the same multiplying factors and converting rule but by only considering strenuous and moderate exercise $(30,31)$. Secondly, in the intervention group, the step number of the first 84 days calculated by the activity tracker (Fitbit Charge HR) was also used to document PA. In order to preserve patient privacy, it was the only data extracted at the end of the study from the Fitbit app to an Excel file (Microsoft, Redmond, WA). Literature shows that step number from a portable monitoring device is a reliable measure to document PA (19-21). In the control group, participants wore pedometers (SW200 Digi-walker, Yamax Inc, Japan) during the first week of the study only to assess their baseline step number. They noted their step number each day and brought the sheet back to the kinesiologist at their first appointment. Thus, pedometers were used as an objective measure to assess PA and sedentarity in the control group for the first week only.

\section{Motivation and autoregulation of PA}

A PA motivation questionnaire was filled at the beginning and at the end of the study by the participants. On a scale of 1 to 7, 1 being strongly disagreeing and 7 being strongly agreeing, participants were invited to rate 16 different motives to exercise regularly. The questionnaire was a validated French translation and cultural adaptation of the Behavioral Regulation in Exercise Questionnaire (BREQ) version 2 (38). The original BREQ questionnaire contains 15 items divided into four categories: (I) external regulation (4 items), (II) introjected regulation (3 items), (III) identified regulation (4 items) and (IV) intrinsic regulation (4 items) (39). The BREQ questionnaire version 2 contains all the same categories and items as the original BREQ but has a supplementary amotivation category composed of 4 items (40). The French version used contained one more 
item about external regulation (38). Since participants had to express minimal motivation to engage in the study, it was decided to remove the amotivation items. According to the self-determination theory (41), external regulation, introjected regulation, identified regulation and intrinsic regulation respectively range in a continuum, the first one being the least self-determined form and the last being the most $(41,42)$. External regulation and introjected regulation can be categorized as a controlled motivation and identified regulation and intrinsic regulation as autonomous motivation $(41,42)$.

\section{Acceptability, satisfaction of the portable monitoring device and compliance}

Satisfaction and acceptability of the portable monitoring device and app were measured by a homemade questionnaire. Participants of the intervention group were invited at the end of the study to fill this 10-question French questionnaire about their satisfaction with the device and the support provided by the research team, their opinion about the information provided by the device and the impact on their lives. The number of days where the portable monitoring device was worn and the compliance were also measured.

\section{Cardiometabolic risk variables}

A prescription for a fasting blood (glycated hemoglobin, lipid profile, kidney function, complete blood count) and a urine test (urine albumin-creatinine ratio) was provided by the physician in charge at baseline and at the end of the study. Hemodynamic measures (resting blood pressure and heart rate) were taken (Welch Allyn spot vital signs LXi, Hill-Rom Holdings Inc, Chicago, IL, USA). Weight and fat percentage were measured with a bioimpedance balance (InBody 520, InBody USA, Cerritos, CA, USA). Height was measured with shoes off with a measuring bar. Weight and height were used for body mass index (BMI) calculation. Waist circumference was measured at the midpoint between the superior iliac crest and the last rib.

\section{Statistical analyses}

A minimum of 500 steps/day was required to include a specific day in the calculation; the cutpoint of a sedentary lifestyle being 5,000 steps, if a participant had less than $90 \%$ of that number, the activity tracker was considered to be only partially worn $(26,43)$. An average weekly step number was calculated to follow the evolution in time (26). In order to obtain a reliable average, a minimum of four days of reliable data in a week was required to include a participant in the calculation of a given week (26). Regarding the motivation questionnaire, each item was analyzed individually then in categories (external, introjected, identified and intrinsic regulation) and in larger classes (controlled and autonomous motivation). The number of days worn was calculated with the Excel step number file: for each day where the step number was higher than zero, the portable monitoring device was considered to be at least partially worn (27). Compliance was calculated by dividing the number of days where the portable monitoring device was worn by the total number of days.

Regarding qualitative data from questionnaires, descriptive statistics were used. Data are expressed as mean \pm standard deviation (SD) unless specified otherwise. Nominal variables were expressed as percentages. For continuous variables, the Student's $t$-test or the Wilcoxon signed-rank test (non normally distributed data) was used to compare characteristics between the control and the intervention group. Fisher's exact test or Chi-squared test was used to verify the equality hypothesis for variables in percentages. Repeated measure of variance analyses were used for parameters with measures available at the beginning and the end of the study. A variability factor between subjects was also considered and treated as a random factor. Another 2-level factor was also defined to take into account measures taken twice in time, time being considered as a repeated measure factor. When evolution comparison of the two groups in time was performed, a third factor with an interactive effect between "group" and "time" was added to the statistical model. Factors with a confounding potential were added if needed. The statistical tests degrees of freedom number was determined with the Kenward-Roger degrees of freedom approximation. Univariate normality hypotheses for continuous variables were verified by the Shapiro-Wilk test on the model residuals transformed by the Cholesky decomposition. Statistical variation tests of Brown and Forsythe were used to check variance homogeneity. Variables were transformed if necessary to satisfy these hypotheses and resulting tests were the ones transcribed. Relationships between variables were reported using the Pearson correlation coefficient. Results were considered statistically significant with a $\alpha \leq 0.05$ level. All these analyses were performed with the software SAS v9.4 (SAS Institute, Cary, NC, USA).

\section{Results}

A total of 48 patients were invited to participate in the 


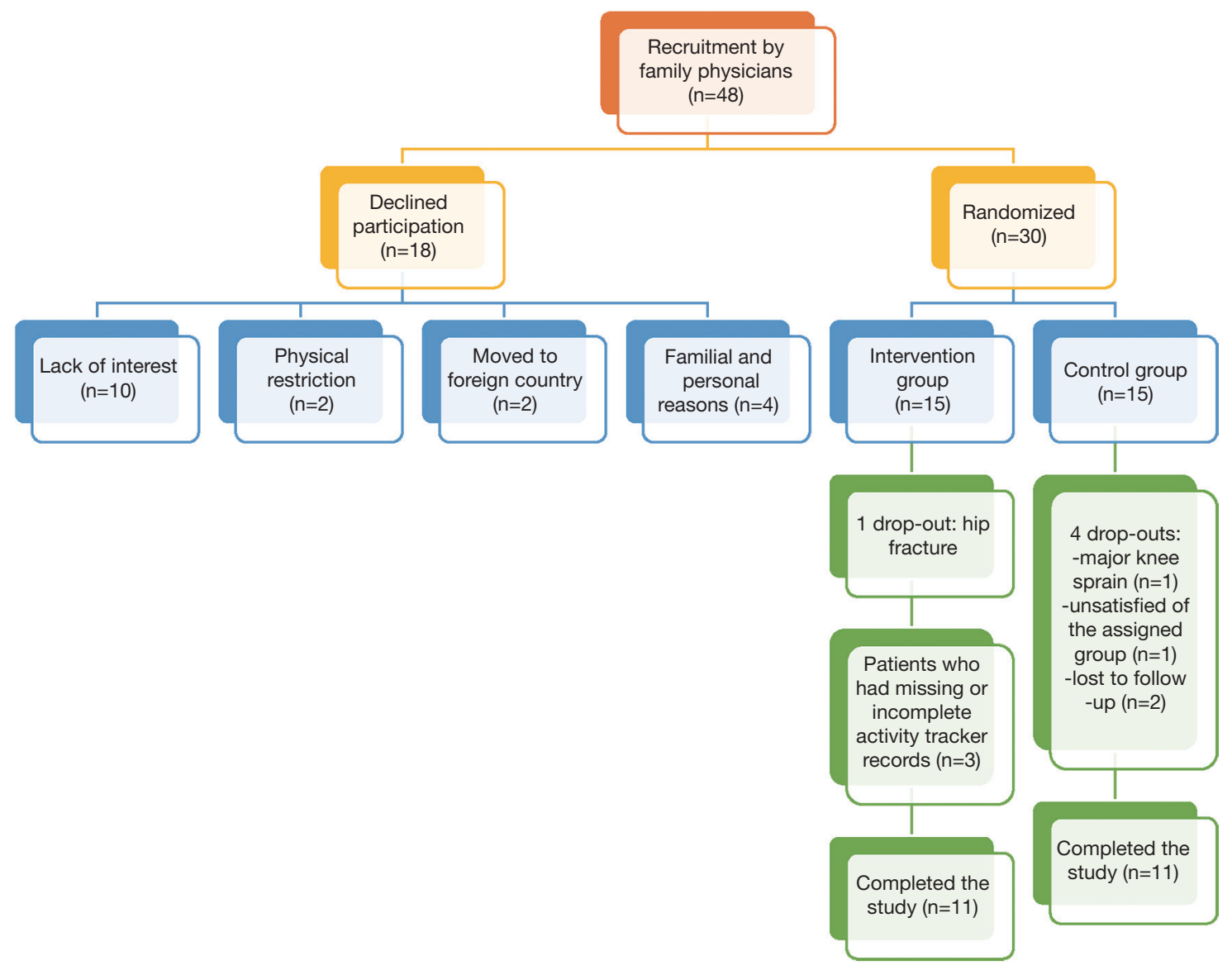

Figure 2 Flowchart of participants in the study.

study by their family physicians between December 2017 and October 2018 (Figure 2). From these, 18 declined participation and 30 were equally assigned randomly between the intervention and the control group. In the intervention group, one participant dropped out because of a hip fracture and there are three cases of missing data from the activity trackers. There were 4 drop-outs in the control group (major knee sprain, unsatisfied of the assigned group, moved away and two lost to follow-up). Therefore, 11 patients completed the 3-month intervention in both groups and were included in the analyses based on their original assigned group. The baseline demographics and clinical characteristics of participants are presented in Table 1.

\section{$P A$ results}

Data of the Godin Leisure-Time Exercise Questionnaire are presented in Table 2. There was no significant difference between groups at baseline. The number of periods a week where a strenuous exercise and a moderate exercise was practiced increased in both groups while the number of periods a week for mild exercise decreased in both groups. For these three items, there was no significant difference between groups and time or time alone. The frequency (often $=1$, sometimes $=2$ or never $/$ rarely $=3$ ) at which a regular training provoked sweat/faster heart rate increased in both groups. The overall score and the score related to health increased in both groups and the differences are significant over time with time P-values of 0.047 and 0.019 respectively.

Table 3 shows the average weekly step numbers as well as the overall step numbers. Baseline step number was $8,162 \pm 3,196$ steps/day in the intervention group. In the intervention group, the highest step average per week was recorded at week $7(8,970 \pm 4,061$ steps/day $)$, after the follow-up phone call scheduled at week 6 . The lowest weekly step average weekly was recorded at week $12(6,752$ $\pm 1,647$ steps/day), at the end of the intervention. Out of 
Table 1 Demographic and cardiometabolic values

\begin{tabular}{|c|c|c|c|c|c|c|}
\hline Baseline and cardiometabolic risk variables & \multicolumn{2}{|c|}{ Control group } & \multicolumn{2}{|c|}{ Intervention group } & P-value & P-value (time \\
\hline Number of participants (n) & 15 & 11 & 15 & $14^{\dagger}$ & - & - \\
\hline Gender (male) & M:8 W:7 & - & M:9 W:6 & - & - & - \\
\hline Age (years) & $65.5 \pm 8.4$ & - & $61.1 \pm 11.0$ & - & - & - \\
\hline Medication use (n) & & & & & - & - \\
\hline Biguanide (metformine) & 11 & 7 & 13 & 12 & - & - \\
\hline GLP-1 therapy & 1 & 0 & 0 & 0 & - & - \\
\hline Sulfonylurea & 1 & 0 & 3 & 4 & - & - \\
\hline Insulin & 3 & 2 & 1 & 1 & - & - \\
\hline No medication & 2 & 2 & 1 & 1 & - & - \\
\hline Weight (kg) & $95.1 \pm 24.2$ & $95.8 \pm 22.9$ & $89.8 \pm 13.1$ & $88.2 \pm 13.4$ & 0.468 & 0.001 \\
\hline Waist circumference $(\mathrm{cm})$ & $112.1 \pm 16.8$ & $113.8 \pm 17.5$ & $105.4 \pm 11.3$ & $104.1 \pm 9.3$ & 0.705 & 0.670 \\
\hline $\mathrm{BMI}\left(\mathrm{kg} / \mathrm{m}^{2}\right)$ & $33.9 \pm 7.5$ & $33.9 \pm 7.5$ & $30.7 \pm 4.0$ & $29.8 \pm 3.7$ & 0.562 & 0.001 \\
\hline Fat percentage (\%) & $40.5 \pm 5.4$ & $41.3 \pm 6.9$ & $35.7 \pm 6.3$ & $33.9 \pm 6.0$ & 0.843 & 0.041 \\
\hline $\mathrm{SBP}(\mathrm{mm} \mathrm{Hg})$ & $137 \pm 16$ & $129 \pm 12$ & $138 \pm 13$ & $127 \pm 14$ & 0.621 & 0.011 \\
\hline $\mathrm{DBP}(\mathrm{mm} \mathrm{Hg})$ & $81 \pm 9$ & $76 \pm 8$ & $79 \pm 8$ & $75 \pm 9$ & 0.781 & 0.046 \\
\hline Total cholesterol (mmol/L) & $4.45 \pm 0.80$ & $4.63 \pm 1.09$ & $4.47 \pm 1.41$ & $4.31 \pm 0.97$ & 0.659 & 0.678 \\
\hline Triglycerides (mmol/L) & $1.76 \pm 0.84$ & $1.63 \pm 0.76$ & $1.74 \pm 0.78$ & $1.34 \pm 0.54$ & 0.210 & 0.107 \\
\hline HDL cholesterol (mmol/L) & $1.53 \pm 0.45$ & $1.35 \pm 0.37$ & $1.26 \pm 0.33$ & $1.35 \pm 0.37$ & 0.014 & 0.852 \\
\hline LDL cholesterol (mmol/L) & $2.12 \pm 0.89$ & $2.53 \pm 1.21$ & $2.41 \pm 1.12$ & $2.35 \pm 0.81$ & 0.678 & 0.796 \\
\hline Non HDL cholesterol & $2.94 \pm 0.91$ & $3.28 \pm 1.29$ & $3.21 \pm 1.38$ & $3.01 \pm 0.92$ & 0.317 & 0.625 \\
\hline Total cholesterol/ HDL cholesterol ratio & $3.13 \pm 1.04$ & $3.69 \pm 1.37$ & $3.69 \pm 1.38$ & $3.35 \pm 0.92$ & 0.103 & 0.582 \\
\hline
\end{tabular}

${ }^{\dagger}$, Only the activity tracker data of 11 participants in the intervention group were extracted. The groups were homogen except for the fat percentage (P-value for groups $=0.022$ ). M, men; W, women; SBP, systolic blood pressure; DBP, diastolic blood pressure; HDL, high-density lipoprotein cholesterol; LDL, low-density lipoprotein cholesterol. 
Table 2 Results of the Godin Leisure-Time Exercise Questionnaire

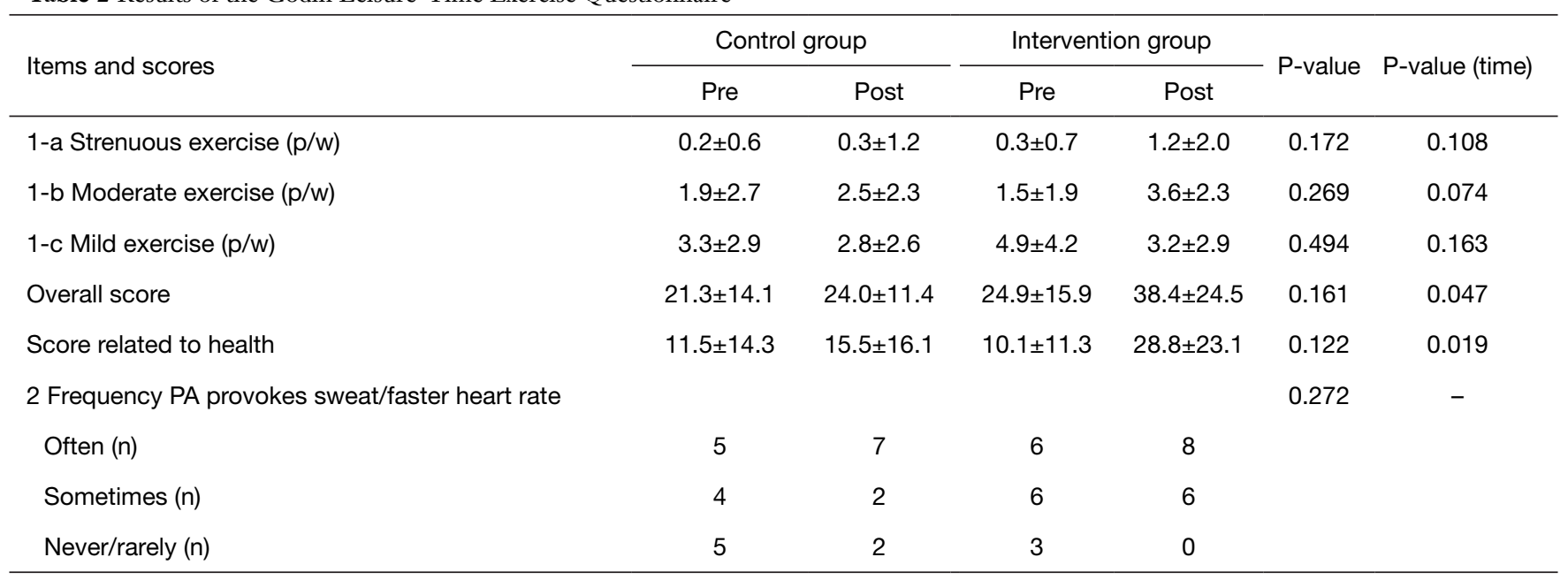

The groups were homogen. $p / w$, periods/week; $n$, number of participants.

Table 3 Step number and confidence interval per week

\begin{tabular}{lccc}
\hline & \multicolumn{3}{c}{ Intervention group } \\
\cline { 2 - 4 } Week & Step number & Confidence interval \\
\cline { 2 - 4 } & & Lower & Upper \\
\hline 1 & $8,162 \pm 3,196$ & 4,831 & 11,173 \\
2 & $8,301 \pm 3,036$ & 5,140 & 11,771 \\
3 & $7,750 \pm 2,853$ & 4,102 & 9,623 \\
4 & $8,370 \pm 3,192$ & 3,651 & 9,678 \\
5 & $7,657 \pm 3,466$ & 3,563 & 11,180 \\
6 & $8,347 \pm 4,312$ & 2,312 & 12,134 \\
7 & $8,970 \pm 4,061$ & 3,772 & 12,011 \\
8 & $7,973 \pm 3,912$ & 3,920 & 8,848 \\
9 & $8,369 \pm 3,569$ & 4,286 & 10,243 \\
10 & $7,525 \pm 3,020$ & 4,366 & 8,908 \\
11 & $7,571 \pm 3,582$ & 3,016 & 8,853 \\
12 & $6,752 \pm 1,647$ & 4,824 & 7,844 \\
Mean & $7,979 \pm 571$ & 7,683 & 8,275 \\
\hline
\end{tabular}

11 participants whose data were extracted, 1 participant walked on average $<5,000$ steps/day, 6 walked on average between 5,000 steps/day and 7,499 steps/day and 4 walked on average $\geq 7,500$ steps/day. Figure 3 presents the daily step average of the 11 participants in the intervention group whose data were extracted.

\section{Motivation and autoregulation of $P A$ results}

Results from the PA motivation questionnaire are presented in Table 4. Overall, the only item with a significant difference between groups and time is "Because my friends/ family/spouse say I should" (external regulation) ( $\mathrm{P}=0.042)$. While the score on a scale of 1 to 7 decreased from $3.0 \pm 1.8$ to $1.9 \pm 1.5$ in the control group, it increased from $2.6 \pm 1.7$ to $3.1 \pm 2.2$ in the intervention group. For the three following items: "Because I value the benefits of exercise", "Because I get restless if I don't exercise regularly" and "Because I get pleasure and satisfaction from participating in exercise", an average score of at least 6 was maintained in both groups. The two first items are categorized as "identified regulation", the category with the highest score in the study (around 6), while the last item is categorized as "intrinsic regulation". Also, the autonomous motivation class scored higher in both groups compared to the controlled motivation class $(\mathrm{P}$ value $<0.001$ ).

\section{Satisfaction and acceptability of the activity tracker and compliance results}

Activity tracker satisfaction and acceptability questionnaire reveals that $86 \%$ of the participants in the intervention group were satisfied or very satisfied of their activity tracker use. Seventy-nine percent of the participant in the intervention group found the information provided by the watch helpful. Participants were asked to select the most useful parameter(s) to keep track of their PA; 


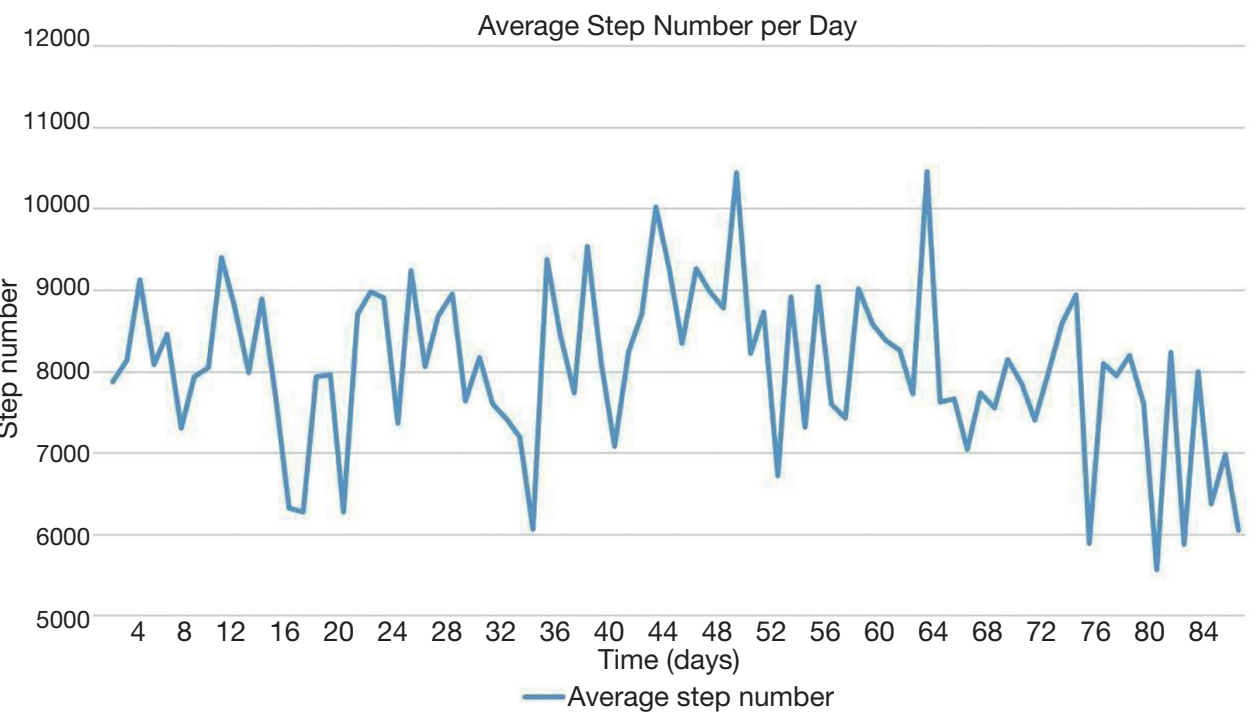

Figure 3 Average of steps per day (measured by the activity tracker in the intervention group).

Table 4 Results of the physical activity motivation questionnaire

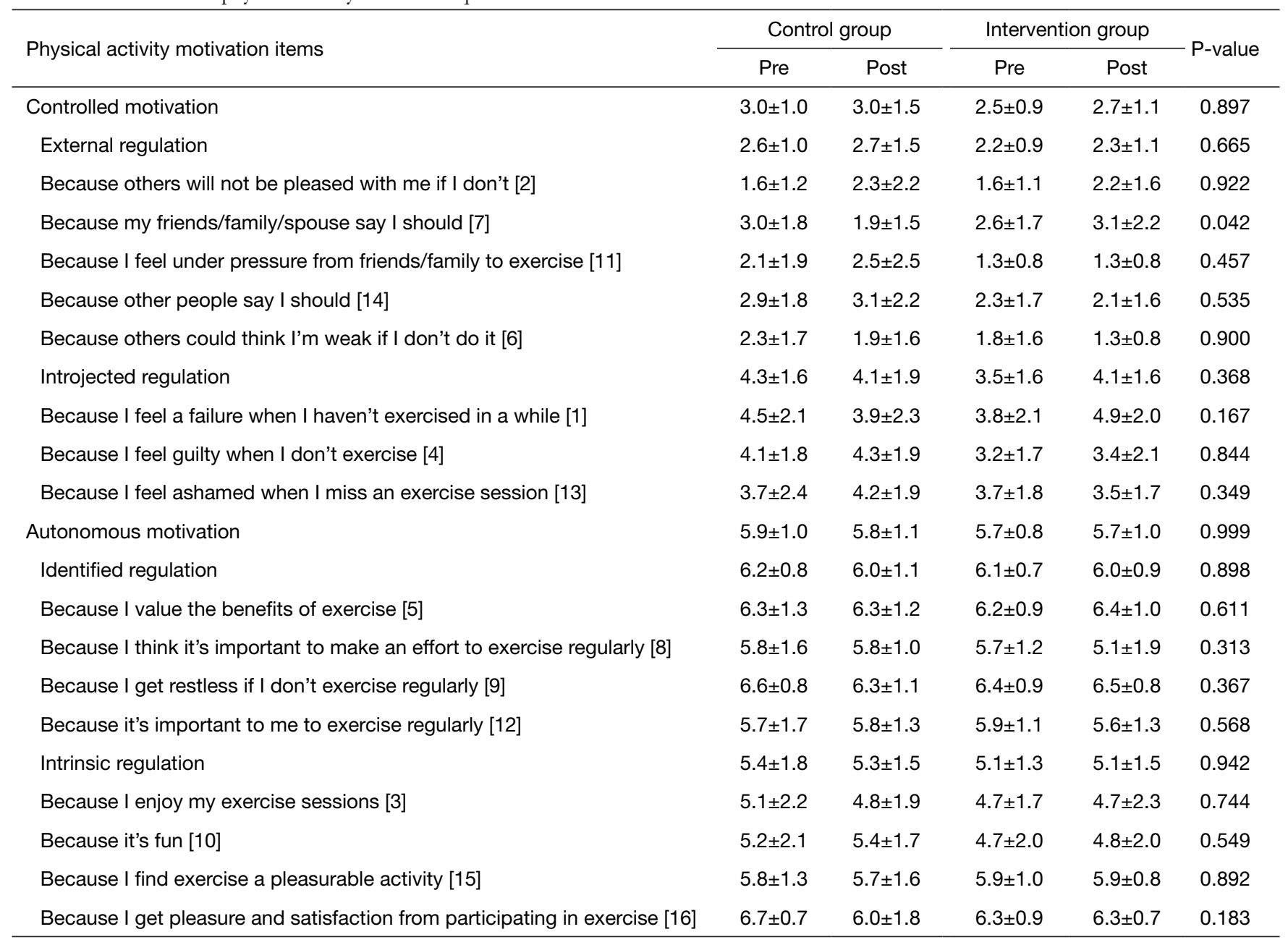


Table 5 Days worn and compliance per participant and per week

\begin{tabular}{lccccc}
\hline Participant & Days worn & Compliance $(\%)$ & Week & Days worn & Compliance $(\%)$ \\
\hline 1 & 84 & 100 & 1 & $6.3 \pm 1.8$ & $90 \pm 26$ \\
2 & 83 & 99 & 2 & $5.9 \pm 2.3$ & $84 \pm 33$ \\
3 & 84 & 100 & 3 & $6.7 \pm 0.6$ & $96 \pm 9$ \\
4 & 84 & 100 & 4 & $6.5 \pm 1.8$ & $92 \pm 26$ \\
5 & 62 & 74 & 5 & $6.9 \pm 0.3$ & $99 \pm 4$ \\
6 & 66 & 79 & 6 & $6.6 \pm 0.9$ & $95 \pm 13$ \\
7 & 84 & 100 & 7 & $6.4 \pm 2.1$ & $91 \pm 30$ \\
8 & 83 & 99 & 8 & $6.3 \pm 2.1$ & $90 \pm 30$ \\
9 & 83 & 99 & 9 & $6.1 \pm 2.1$ & $87 \pm 30$ \\
10 & 48 & 57 & 10 & $5.6 \pm 2.8$ & $81 \pm 40$ \\
11 & 60 & 71 & 11 & $5.5 \pm 2.5$ & $78 \pm 36$ \\
12 & - & - & 12 & $5.9 \pm 2.2$ & $84 \pm 31$ \\
13 & - & - & - & - & - \\
14 & - & - & - & - & - \\
15 & - & - & - & - & - \\
Mean & $75 \pm 13$ & $89 \pm 16$ & - & $6.2 \pm 0.4$ & $89 \pm 6$ \\
\hline
\end{tabular}

the step number was mentioned by 6 participants, while weight, distance and sedentary and active minutes were mentioned by 3 each and calories were mentioned once. For the most useful parameter(s) to motivate to practice PA, the step number was selected 5 times, the weight 4 times, the distance 3 times, the sedentary and active minutes 2 times and the calories 1 time. Seventy-five percent of the participants considered that the activity tracker incites them to stick to their PA program after the end of the intervention. Fifty percent of the participants were planning to buy an activity tracker after the study. Table 5 presents the days worn and the compliance per participant and per week. Out of the 11 participants whose data were extracted in the intervention group, 7 participants wore the activity tracker 83 or 84 days out of 84 . Participants wore on average the activity tracker $75 \% \pm 13 \%$ (95\% CI: $67-82 \%$ ) days out of 84 and $6.2 \pm 0.4$ days weekly (95\% CI: 5.6-6.8 days/week). The average compliance among participants was $89 \% \pm 16 \%$ (95\% CI: 80-97\%).

\section{Cardiometabolic risk variable results}

As shown in Table 1, there was no significant difference between groups at baseline except for fat percentage $(\mathrm{P}=0.022)$ which was higher $(4.8 \%)$ in the control group. Weight, BMI and fat percentage increased in the control group and decreased in the intervention group with a significant difference over time (time P-values $=0.001$, 0.001 and 0.041 respectively). Systolic blood pressure (SBP) and diastolic blood pressure (DBP) decreased over time in both groups (P-values $=0.011$ and 0.046 respectively). High-density lipoprotein (HDL) cholesterol increased in the intervention group and decreased in the control group. This difference in HDL cholesterol is significant between groups and time $(\mathrm{P}=0.014)$. Multivariate regression analysis between average daily steps (of the first week in pre and of the following weeks in post) and changes in cardiometabolic risk variables showed significant correlations in pre and post-intervention regarding waist circumference (pre: $-0.721, \mathrm{P}=0.044$; post: $-0.736, \mathrm{P}=0.038$ ), BMI (pre: -0.764 , $\mathrm{P}=0.010$; post: $-0.771, \mathrm{P}=0.009$ ) and fat percentage (pre: $-0.654, \mathrm{P}=0.040$; post: $-0.686, \mathrm{P}=0.028$ ) in the intervention group. There was no significant correlations in the control group. When pooling both groups, we observed significant correlations between changes in weight and BMI with changes in glycated hemoglobin $(0.681, \mathrm{P}<0.001$ and 0.702 , 
$\mathrm{P}<0.001$ respectively).

\section{Discussion}

Results of the present randomized controlled pilot study showed that the overall score and score related to health from the Godin Leisure-Time Exercise Questionnaire significantly increased in both groups, the increment being higher in the intervention group. The autonomous motivation was significantly higher than the controlled motivation, demonstrating that participants in both groups exercised more for the benefits of PA and the pleasure of practicing PA. Eighty-six percent of patients in the intervention group were satisfied of their activity tracker use and the compliance remained high during the study. Our multivariate regression analysis between steps and changes in cardiometabolic risk variables showed significant correlations in pre and post-intervention for waist circumference, BMI and fat percentage in the intervention group. We found that HDL cholesterol significantly increased in the intervention group while it decreased in the control group. Resting SBP and DBP significantly decreased over time in both groups. Glycated hemoglobin tended to decrease in both groups. Few studies have been conducted on activity trackers like the Fitbit watch since it is a newer technology and, to the best of our knowledge, no study included a kinesiologistled PA intervention. Moreover, this study was conducted in a primary care setting and the outcome was oriented on the satisfaction of the activity tracker, the motivation to practice $\mathrm{PA}$ and cardiometabolic variables. Our study documents for the first time the motivation as assessed by the BREQ questionnaire version 2 in an activity tracker intervention in patients with T2D.

Regarding the PA findings, participants in the control group went from "moderately active" overall and "insufficiently active" in terms of health benefits to respectively "active" and "moderately active", while those in the intervention group remained "active" overall and went from "insufficiently active" to "active" in terms of health benefits $(30,31)$. The PA promotion intervention supported by a kinesiologist and the personalized training plan in both groups could explain these increases. Indeed, participants in both groups were more aware of the PA intensity to reach and had a PA plan to follow. We believe that the higher increment in the intervention group, although not significant probably due to a lack of power, could be explained by an increased motivation for PA due to the activity tracker.
Regarding the step numbers, the highest average step number per week was recorded at week 7 , just after the follow-up phone call with the kinesiologist, which demonstrates the positive impact this health care professional had. The overall average in the intervention group was $>7,500$ steps/day and thus can be interpreted as a physically active lifestyle meeting the moderate to vigorous physical activity (MVPA) recommendations, although there were a lot of interindividual variabilities (43). A study conducted by Houle et al. revealed that targeting 7500 steps/day can improve cardiovascular risk factors during the first year following an acute coronary syndrome which represents a realistic goal (44). In the Netherlands, Kooiman et al. tested the efficacy of a 13-week activity tracker (Fitbit Zip) based intervention with an online selftracking program on PA, glycated hemoglobin and other health measures in 72 patients with T2D (26). Kooiman et al. used the steps/day substantial variability to explain the absence of significant overall decline in glycated hemoglobin and a similar phenomenon likely occurred in the present study (26). Indeed, $36 \%$ of the intervention group participants met 7,500 steps/day recommendation while $64 \%$ did not, in comparison of respectively $39 \%$ and $61 \%$ in the Kooiman study (26). In the United States, Polgreen et al. compared three groups of patients with T2D or prediabetes- Fitbit only (48 participants), Fitbit with reminders (44 participants) and Fitbit with both reminders and goal setting (46 participants) to document the impact of automatic text-message reminders on Fitbit adherence and PA on a 6-month period (27). The Fitbit only group had the highest average daily steps with 7,123 steps, which is similar to the average of $7,979 \pm 572$ steps/day observed in our study.

Looking at motivation and autoregulation of PA data, the three items that scored the highest are two identified regulation items and one intrinsic regulation item. Autonomous motivation class (comprising identified regulation and intrinsic regulation) scored significantly higher than controlled motivation class in both groups. Therefore, participants in both groups were more likely to exercise because they valued the benefits of exercise, it made them feel better and they got pleasure and satisfaction from it. Literature shows that autonomous forms of motivation are more correlated with PA and identified regulation would predict initial/short-term PA while intrinsic motivation would predict more long-term PA adherence (45).

Eighty-six percent of the participants in the intervention group were satisfied or very satisfied of their activity tracker use which is comparable to the Canadian population owning 
at least one connected health object (83\%) (46). The step number was considered as the most helpful parameter to keep track of PA and to motivate to practice PA, which is interesting since this information could also be displayed by a pedometer, a cheaper device. Seventy-five percent of the participants considered that the activity tracker will incite them to stick to their PA program after the end of the intervention. This important proportion shows the impact an activity tracker can have on long-term motivation. Also, $50 \%$ of the participants were planning to buy an activity tracker after the study which is also more than the general Canadian population (15\% enough likely and $1 \%$ really likely) (46). On the other hand, one must keep in mind that our population included patients with T2D. The compliance was high and remained stable along time, showing in an objective way the appreciation of wearing and using the device.

Up to now, few studies have demonstrated the impact of PA supported by an activity tracker on improvement of cardiometabolic parameters. We found that HDL cholesterol levels significantly increased in the intervention group and decreased in the control group. Resting SBP and DBP significantly decreased over time in both groups. Hayashino et al. reported in their meta-analysis documenting the effects of supervised exercise in patients with T2D, an increase of HDL cholesterol level and decrease of SBP and DBP after exercise (47). In our study, a lower waist circumference, $\mathrm{BMI}$ and fat percentage were significantly associated with a higher daily step number in the intervention group, in accordance with the existing literature $(48,49)$. The nonstatistically significant glycated hemoglobin decrease in both groups can be explained by the small sample size and the short study duration. In a meta-analysis documenting the impact of accelerometers and pedometers in patients with T2D, Baskerville et al. reported no significant difference in glycated hemoglobin, BMI, blood pressure and lipid profile (22). In accordance, Qiu et al. reported in their meta-analysis regarding pedometers that there was no evidence that such portable monitoring devices alone could improve glycemic control (25). Of note, no study included a kinesiologist-led PA intervention. Kooiman et al. reported no overall difference over 13 weeks in glycated hemoglobin, but a subgroup analysis revealed that "responders" (patients who increased their PA by at least 1,000 steps/day compared to baseline) had a significant decrease in glycated hemoglobin over time (26).

This study had limitations and strengths. The first limitation was the limited number of activity trackers.
Also, the activity tracker data of four candidates were not extracted. One participant had forgotten his login access, another abandoned and two technical problems impeded access to the other participants' data. The fact that the participants had absolute control over their data sharing complicated data extraction. It might have been easier if the participants' login accesses were given by the research team so they could have direct access to the data with the participants' authorization. Some participants needed more support to be able to correctly use the activity tracker and some technical problems occurred. A strength was that the study was initiated by a patient partner. He gave his layperson opinion on the way cardiometabolic risk variables and other measurements should be taken which gave credibility to the study. Also, the study used a technology that is widely available and is therefore an interesting avenue for easy implementation in the community. The study was conducted in a primary care center in a real life context, which also provides evidence on its feasibility.

\section{Conclusions}

In conclusion, our pilot study shows that an activity tracker could be a potential motivation tool to increase $\mathrm{PA}$ in patients with T2D. Also, the use of an activity tracker improves some cardiometabolic risk variables. The implantation of activity trackers in a primary care setting is feasible and these devices could become an additional tool in the management of chronic diseases. A larger scale study is required to assess the effectiveness of an activity tracker on cardiometabolic risk variables and PA. The potentialisation of such an approach using a kinesiologistled PA intervention need further studies.

\section{Acknowledgments}

We would like to thank participants, general practitioners and all the staff of GMF-U Quatre-Bourgeois and also of the Institut universitaire de cardiologie et de pneumologie de Québec.

Funding: This work was supported by program grants from VITAM-Centre de recherche en santé durable.

\section{Footnote}

Reporting Checklist: The authors have completed the CONSORT reporting checklist. Available at http://dx.doi. org/10.21037/mhealth-20-154 
Data Sharing Statement: Available at http://dx.doi. org/10.21037/mhealth-20-154

Peer Review File: Available at http://dx.doi.org/10.21037/ mhealth-20-154

Conflicts of Interest: All authors have completed the ICMJE uniform disclosure form (available at http://dx.doi. org/10.21037/mhealth-20-154). Dr. JPD reports grants from Canadian Institutes of Health Research, outside the submitted work. The other authors have no conflicts of interest to declare.

Ethical Statement: The authors are accountable for all aspects of the work in ensuring that questions related to the accuracy or integrity of any part of the work are appropriately investigated and resolved. The trial was conducted in accordance with the Declaration of Helsinki (as revised in 2013). The study was approved by institutional ethics board of Centre intégré universitaire en santé et services sociaux (CIUSSS) de la Capitale-Nationale (NO.: 2017-2018-07) and informed consent was taken from all the participants.

Open Access Statement: This is an Open Access article distributed in accordance with the Creative Commons Attribution-NonCommercial-NoDerivs 4.0 International License (CC BY-NC-ND 4.0), which permits the noncommercial replication and distribution of the article with the strict proviso that no changes or edits are made and the original work is properly cited (including links to both the formal publication through the relevant DOI and the license). See: https://creativecommons.org/licenses/by-nc-nd/4.0/.

\section{References}

1. Diabetes. World Health Organization. 2020. Available online: https://www.who.int/news-room/fact-sheets/detail/ diabetes [accessed 2020-06-22]

2. Diabetes Facts and Figures. International Diabetes Federation. Available online: https://www.idf.org/ aboutdiabetes/what-is-diabetes/facts-figures.html [accessed 2020-06-22]

3. Le diabète en chiffres. Diabète Québec. Available online: https://www.diabete.qc.ca/fr/diabete-quebec/a-propos/ salle-de-presse/le-diabete-en-chiffres/

4. Lee IM, Shiroma EJ, Lobelo F, et al. Effect of physical inactivity on major non-communicable diseases worldwide: an analysis of burden of disease and life expectancy. Lancet 2012;380:219-29.

5. Sanz C, Gautier JF, Hanaire H. Physical exercise for the prevention and treatment of type 2 diabetes. Diabetes Metab 2010;36:346-51.

6. Thomas DE, Elliott EJ, Naughton GA. Exercise for type 2 diabetes mellitus. Cochrane Database Syst Rev 2006: (3):CD002968.

7. Look AHEAD Research Group, Wing RR, Bolin P, et al. Cardiovascular Effects of Intensive Lifestyle Intervention in Type 2 Diabetes. N Engl J Med 2013;369:145-54.

8. Chen L, Pei JH, Kuang J, et al. Effect of lifestyle intervention in patients with type 2 diabetes: a metaanalysis. Metabolism 2015;64:338-47.

9. Snowling NJ, Hopkins WG. Effects of different modes of exercise training on glucose control and risk factors for complications in type 2 diabetic patients: a meta-analysis. Diabetes Care 2006;29:2518-27.

10. Myers VH, McVay MA, Brashear MM, et al. Exercise training and quality of life in individuals with type 2 diabetes: a randomized controlled trial. Diabetes Care 2013;36:1884-90.

11. L'activité physique en quelques chiffres: Ministère de la Santé et des Services Sociaux; 2018 [updated Nov 2018. Available online: https://www.msss.gouv.qc.ca/ professionnels/statistiques-donnees-sante-bien-etre/flashsurveillance/activite-physique-en-quelques-chiffres/

12. Feter N, Dos Santos TS, Caputo EL, et al. What is the role of smartphones on physical activity promotion? A systematic review and meta-analysis. Int J Public Health 2019;64:679-90.

13. Schoeppe S, Alley S, Van Lippevelde W, et al. Efficacy of interventions that use apps to improve diet, physical activity and sedentary behaviour: a systematic review. Int J Behav Nutr Phys Act 2016;13:127.

14. Romeo A, Edney S, Plotnikoff R, et al. Can Smartphone Apps Increase Physical Activity? Systematic Review and Meta-Analysis. J Med Internet Res 2019;21:e12053.

15. Afshin A, Babalola D, Mclean M, et al. Information Technology and Lifestyle: A Systematic Evaluation of Internet and Mobile Interventions for Improving Diet, Physical Activity, Obesity, Tobacco, and Alcohol Use. J Am Heart Assoc 2016;5:e003058.

16. Bravata DM, Smith-Spangler C, Sundaram V, et al. Using pedometers to increase physical activity and improve health: a systematic review. JAMA 2007;298:2296-304.

17. Brickwood KJ, Watson G, O'Brien J, et al. ConsumerBased Wearable Activity Trackers Increase Physical 
Activity Participation: Systematic Review and MetaAnalysis. JMIR Mhealth Uhealth 2019;7:e11819.

18. Coughlin SS, Stewart J. Use of Consumer Wearable Devices to Promote Physical Activity: A Review of Health Intervention Studies. J Environ Health Sci 2016. doi:10.15436/2378-6841.16.1123.

19. Evenson KR, Goto MM, Furberg RD. Systematic review of the validity and reliability of consumer-wearable activity trackers. Int J Behav Nutr Phys Act 2015;12:159.

20. Weatherall J, Paprocki Y, Meyer TM, et al. Sleep Tracking and Exercise in Patients With Type 2 Diabetes Mellitus (Step-D): Pilot Study to Determine Correlations Between Fitbit Data and Patient-Reported Outcomes. JMIR Mhealth Uhealth 2018;6:e131.

21. Straiton N, Alharbi M, Bauman A, et al. The validity and reliability of consumer-grade activity trackers in older, community-dwelling adults: A systematic review. Maturitas 2018;112:85-93.

22. Baskerville R, Ricci-Cabello I, Roberts N, et al. Impact of accelerometer and pedometer use on physical activity and glycaemic control in people with Type 2 diabetes: a systematic review and meta-analysis. Diabet Med 2017;34:612-20.

23. Franssen WMA, Franssen G, Spaas J, et al. Can consumer wearable activity tracker-based interventions improve physical activity and cardiometabolic health in patients with chronic diseases? A systematic review and metaanalysis of randomised controlled trials. Int J Behav Nutr Phys Act 2020;17:57.

24. Kirk MA, Amiri M, Pirbaglou M, et al. Wearable Technology and Physical Activity Behavior Change in Adults With Chronic Cardiometabolic Disease: A Systematic Review and Meta-Analysis. Am J Health Promot 2019;33:778-91.

25. Qiu S, Cai X, Chen X, et al. Step counter use in type 2 diabetes: a meta-analysis of randomized controlled trials. BMC Med 2014;12:36.

26. Kooiman TJM, de Groot M, Hoogenberg K, et al. Selftracking of Physical Activity in People With Type 2 Diabetes: A Randomized Controlled Trial. Comput Inform Nurs 2018;36:340-9.

27. Polgreen LA, Anthony C, Carr L, et al. The effect of automated text messaging and goal setting on pedometer adherence and physical activity in patients with diabetes: A randomized controlled trial. PLoS One 2018;13:e0195797.

28. Houle J, Doyon O, Vadeboncoeur N, et al. Innovative program to increase physical activity following an acute coronary syndrome: randomized controlled trial. Patient
Educ Couns 2011;85:e237-44.

29. Houle J, Doyon O, Vadeboncoeur N, et al. Effectiveness of a pedometer-based program using a socio-cognitive intervention on physical activity and quality of life in a setting of cardiac rehabilitation. Can J Cardiol 2012;28:27-32.

30. Godin G, Shephard RJ. Leisure-Time Exercise Questionnaire. Medicine and Science in Sports and Exercise 1997;29:S36-8.

31. Godin G. The Godin-Shephard Leisure-Time Physical Activity Questionnaire. Health and Fitness Journal of Canada 2011;4:18-22.

32. Miquelon P, Castonguay A. Motives for Participation in Physical Activity and Observance of Physical Activity Recommendations among Adults with Type 2 Diabetes. Can J Diabetes 2016;40:399-405.

33. Canadian Physical Activity Guidelines. Canadian Society for Exercise Physiology. Available online: https:// csepguidelines.ca/wp-content/uploads/2018/03/CSEP_ PAGuidelines_adults_en.pdf

34. World Medical Association Declaration of Helsinki: ethical principles for medical research involving human subjects. Jama 2013;310:2191-4.

35. Rhéaume C, Gagnon MP, Poirier P, et al. Portable Monitoring Device, Physical Activity Motivation and Patients With Type 2 Diabetes (DBFitbit). ClinicalTrials. gov. October 17, 2018. Available online: https:// clinicaltrials.gov/ct2/show/study/NCT03709966

36. Amireault S, Godin G. The Godin-Shephard leisuretime physical activity questionnaire: validity evidence supporting its use for classifying healthy adults into active and insufficiently active categories. Percept Mot Skills 2015;120:604-22.

37. Pereira MA, FitzerGerald SJ, Gregg EW, et al. A collection of Physical Activity Questionnaires for healthrelated research. Med Sci Sports Exerc 1997;29:S1-205.

38. Miquelon P, Castonguay A. Comment la régulation intégrée contribue-t-elle au maintien de la pratique de l'activité physique? 84e Congrès de l'ACFAS; Montreal: 2016.

39. Mullan E, Markland D, Ingledew DK. A graded conceptualisation of self-determination in the regulation of exercise behaviour: Development of a measure using confirmatory factor analytic procedures. Pers Individ Dif 1997;23:745-52.

40. Markland D, Tobin V. A Modification to the Behavioural Regulation in Exercise Questionnaire to Include an Assessment of Amotivation. J Sport Exerc Psychol 2004;26:191-6. 
41. Deci E, Ryan R. The "What" and "Why" of Goal Pursuits: Human Needs and the Self-Determination of Behavior. Psychological Inquiry 2000;11:227-68.

42. Deci EL, Ryan RM. Self-determination theory: A macrotheory of human motivation, development, and health. Can Psychol 2008;49:182-5.

43. Tudor-Locke C, Craig CL, Thyfault JP, et al. A stepdefined sedentary lifestyle index: $<5000$ steps/day. Appl Physiol Nutr Metab 2013;38:100-14.

44. Houle J, Valera B, Gaudet-Savard T, et al. Daily steps threshold to improve cardiovascular disease risk factors during the year after an acute coronary syndrome. J Cardiopulm Rehabil Prev 2013;33:406-10.

45. Teixeira PJ, Carraça EV, Markland D, et al. Exercise, physical activity, and self-determination theory: A systematic review. Int J Behav Nutr Phys Act 2012;9:78.

doi: $10.21037 /$ mhealth-20-154

Cite this article as: Pelletier C, Gagnon MP, Alméras N, Després JP, Poirier P, Tremblay A, Chabot C, Rhéaume C. Using an activity tracker to increase motivation for physical activity in patients with type 2 diabetes in primary care: a randomized pilot trial. mHealth 2021;7:59.
46. Paré G, Bourget C, Aguirre M, et al. Diffusion de la santé connectée au Canada. Montréal, Québec (Canada): CEFRIO2017.

47. Hayashino Y, Jackson JL, Fukumori N, et al. Effects of supervised exercise on lipid profiles and blood pressure control in people with type 2 diabetes mellitus: a metaanalysis of randomized controlled trials. Diabetes Res Clin Pract 2012;98:349-60.

48. Baillot A, Romain AJ, Boisvert-Vigneault K, et al. Effects of lifestyle interventions that include a physical activity component in class II and III obese individuals: a systematic review and meta-analysis. PLoS One 2015;10:e0119017.

49. National Heart L, and Blood Institute. Metabolic Syndrome. Available online: https://www.nhlbi.nih.gov/ health-topics/metabolic-syndrome [accessed 2020-08-06] 